\title{
Compactação de solo cultivado com cana-de-açúcar em Baía Formosa, Rio Grande do Norte $^{1}$
}

\author{
Francisco Xavier de Oliveira Filho ${ }^{2 *}$, Neyton de Oliveira Miranda ${ }^{2}$, José Francismar de Medeiros ${ }^{2}$, \\ Paulo César Moura da Silva², Francisco de Oliveira Mesquita², Thiago Kennedy Gomes Costa ${ }^{3}$
}

$10.1590 / 0034-737 X 201663050017$

\section{RESUMO}

A compactação do solo é uma das principais limitações impostas ao desenvolvimento do sistema radicular da canade-açúcar, promovendo a redução da capacidade de absorção de água e nutrientes pela planta. Neste contexto, objetivouse identificar regiões no campo com diferentes níveis de compactação, de modo a permitir a adoção de manejo localizado. O trabalho foi realizado na Usina Vale Verde, em Baía Formosa-RN, Brasil, onde a resistência do solo à penetração (RSP) foi avaliada em área de 113,59 ha, dividida em 12 talhões cultivados com cana-de-açúcar, onde foram locados 113 pontos georeferenciados. As determinações foram feitas nas camadas de 0-10, 11-20, 21-30 e 31-40 cm de profundidade, utilizando-se penetrômetro com sistema automático de medição. Foram utilizadas ferramentas de geoestatística para avaliar a dependência espacial das variáveis e confeccionar, por meio da interpolação por krigagem, mapas de isovalores da RSP. As figuras de isovalores localizam no campo áreas com diferentes níveis de compactação do solo. A descompactação do solo pode ser evitada em $18,80 \%$ da área total, principalmente nos talhões 45 e 48 . Nas áreas que necessitam descompactação, esta deve ser realizada a profundidade maior do que $40 \mathrm{~cm}$.

Palavras-chave: geoestatística; penetrômetro; resistência do solo à penetração.

\section{ABSTRACT}

\section{Soil compaction in a sugarcane field at Baía Formosa, Rio Grande do Norte, Brazil}

Soil compaction is one of the main constraints imposed to growth of sugar cane root system, with consequences as reduction on plant ability to absorb water and nutrients. In this context, this study had as objective to identify regions in the field with different compaction levels, allowing the adoption of site specific management. The work was carried out at the Green Valley Sugarcane Mill, in Baía Formosa, RN, Brazil. Soil penetration resistance (RSP) was used as indicative parameter of soil compaction, and was assessed in an area of 113.59 ha, divided in 12 sugarcane fields, where 113 georeferenced points were located. Determinations were made in the 0-10, 11-20, 21-30 and 31-40 cm depths, by means of a soil penetrometer with automatic data acquisition system. Data were analyzed through descriptive statistics and geostatistical tools, which were used for evaluating spatial dependence of variables and draw contour maps, after kriging interpolation. Contour maps show regions in the area with different soil compaction levels. Subsoiling may be avoided in $18.80 \%$ of the total area, mainly in the fields 45 and 48 . In other regions subsoiling must be performed deeper than $40 \mathrm{~cm}$.

Key words: geostatistics; soil penetrometer; soil penetration resistance.

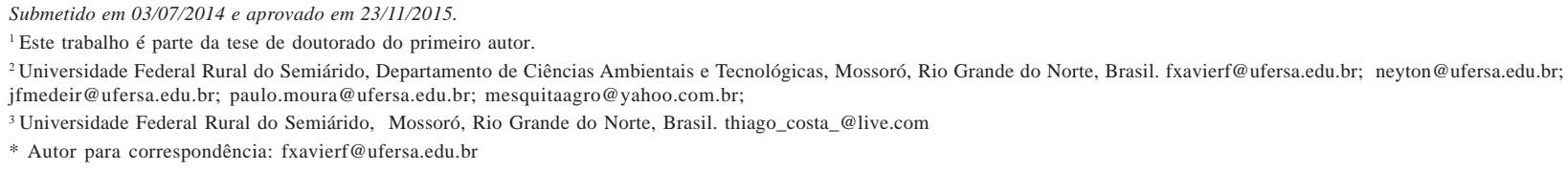




\section{INTRODUÇÃO}

A cana-de-açúcar (Saccharum offcinarum L.) tem importância agrícola e industrial no Brasil, devido ao valor da produção, área explorada e suas múltiplas utilidades. O país é o maior produtor mundial de açúcar e de etanol, um biocombustível importante, econômica e ambientalmente (Rodrigues et al., 2010). No Rio Grande do Norte, o setor canavieiro é a principal atividade agrícola em área plantada e receita gerada, que é de $\mathrm{R} \$ 271.000 .000$. A produtividade da cana-de-açúcar no estado (59 t ha ${ }^{-1}$ ) é $16 \%$ menor do que a média do país e semelhante à média da região nordeste (IBGE, 2014).

Atualmente, com o incremento das áreas agrícolas, há maior preocupação com os problemas relacionados à compactação do solo resultante das operações mecanizadas. A degradação cumulativa da qualidade física do solo, ao longo dos anos de cultivo se reflete nas propriedades do solo relacionadas à compactação, independentemente do sistema de manejo da cana-de-açúcar (Roque et al., 2010). O impacto sobre a produtividade das culturas devese a influencia da compactação sobre as demais propriedades físicas do solo podendo afetar as plantas, da germinação ao desenvolvimento radicular e aéreo (Reichert et al., 2007).

A resistência mecânica à penetração (RSP) é muito usada para indicar o grau de compactação, por ser relacionada com diversos atributos do solo indicativos desse tipo de degradação. Os equipamentos utilizados para esta determinação, os penetrômetros, medem a resistência oferecida pelo solo à penetração de uma ponta cônica (Molin et al., 2012). Os autores ressaltam que o teor de água e a textura do solo são os fatores que mais influenciam na magnitude das medidas de resistência à penetração.

A cultura da cana-de-açúcar tem na parte aérea o seu produto comercial, os colmos, e um sistema radicular formado por rizomas e raízes fasciculadas, das quais $85 \%$ estão localizadas até $50 \mathrm{~cm}$ de profundidade (Silva \& Silva, 2012). Por isso, é muito afetada pela compactação do solo, agravada pelas várias operações mecanizadas, como a colheita, a qual, segundo relato de Lima et al. (2013b), causa redução acima de $50 \%$ do volume de macroporos do solo, sendo o valor de macroporosidade de $10 \%$ considerado crítico para o crescimento das plantas (Reichert et al., 2007).

A operação de subsolagem, a mais usada para romper camadas de solo que sofreram compactação e minimizar seus efeitos, em geral é realizada no campo todo. Ela exige alto consumo de energia e apresenta custo elevado, devendo ser realizada de forma localizada, quando houver camada impeditiva ao fluxo de água e ao desenvolvimento de raízes e quando a resistência à penetração for maior ou igual a um valor crítico, além de ser conhecida a localização e espessura da camada compactada (Silva et al.,
2004). Para isso, devem ser empregados mapas que representem a intensidade de compactação e a variação na profundidade do preparo do solo, ao longo do campo (Gorucu etal., 2006).

A adoção do manejo localizado pressupõe a divisão do campo em classes homogêneas, que recebem o mesmo tratamento ou dose. Os critérios utilizados são a produtividade e os atributos químicos e físicos do solo, entre os quais a RSP e a textura. Os custos para dividir o campo em classes de manejo compensam, se houver diferenças na resposta agronômica entre elas e praticabilidade do manejo variável (Taylor et al., 2007; Bazzi et al., 2013).

A especificação de uma profundidade adequada para se realizar a subsolagem pode ser feita por diferentes critérios. Se o critério adotado for o valor máximo de RSP, existe a possibilidade de que a camada compactada não seja removida completamente (Silva et al., 2004); se a subsolagem for baseada na profundidade de ocorrência da máxima RSP, pode-se concluir pela sua inviabilidade, no caso de grande profundidade. A maneira mais adequada seria estipular um valor crítico de resistência à penetração, limitante para a cultura, que em conjunto com o gráfico de RSP localiza no perfil a camada compactada e determina sua espessura.

A adoção de valor crítico de resistência à penetração, relatada por Silva et al. (2004), permite determinar o volume de solo a ser mobilizado e decidir sobre a realização da subsolagem. Para isso, os métodos utilizados são o probabilístico e suas técnicas estatísticas, o determinístico, que calcula a proporção do volume de solo mobilizado e sua distribuição espacial, e o geoestatístico, que permite elaborar mapas de isovalores para delinear zonas de manejo e subsolar apenas onde necessário.

O manejo localizado da subsolagem é vantajoso por sua realização na profundidade mais adequada e em locais onde seja realmente necessária, gerando economia de energia e de custos com tratores, combustível, tempo e desgaste de máquinas (Silva et al., 2004; Salvador et al., 2008). Segundo Bianchini et al. (1999), ocorre aumento de forma quadrática da força necessária, em função da profundidade de subsolagem. Economia de combustível entre 27 e $43 \%$ foi obtida por Raper et al. (2007), quando a subsolagem foi realizada de forma variável, conforme a camada de solo a ser descompactada, e de 33,8\%, por Gorucu Keskin et al. (2011), por terem subsolado 67 a $75 \%$ dos campos em profundidade menor do que a normalmente usada.

A realização da subsolagem apenas nas regiões do campo onde seja necessária e na profundidade mais adequada pode reduzir o tempo necessário e os custos da operação. Neste sentido, este trabalho visou a determinar a resistência do solo à penetração, de forma georreferenciada, em áreas sob cultivo de cana-de-açúcar, para localizar regiões do campo com diferentes níveis de compactação. 


\section{MATERIAL E MÉTODOS}

O trabalho foi desenvolvido nos anos de 2012 e 2013 na Usina Vale Verde, em Baía Formosa, RN, localizada nas coordenadas de 6 $6^{\circ} 22^{\prime} 23^{\prime \prime} \mathrm{S} \mathrm{e} 35^{\circ} 00^{\prime} 34^{\prime \prime}$ O. O clima local é do tipo As, pela classificação de Köppen-Geiger, ou seja, tropical chuvoso com uma estação seca e um período chuvoso, entre janeiro e agosto, temperatura média anual de $27,5^{\circ} \mathrm{C}$ e pluviosidade média de $1.625 \mathrm{~mm}^{2} \mathrm{no}^{-1}$.

A área de estudo, com 113,59 ha, está situada na Fazenda Casqueira (Figura 1A), cultivada com cana-de-açúcar e subdividida em 12 talhões (Figura 1B). A malha amostral foi composta por 113 pontos distribuídos regularmente, distantes $50 \mathrm{~m}$ a partir da borda do perímetro e $100 \mathrm{~m}$ entre eles (Tabela 1). Esses pontos foram georreferenciados em coordenadas UTM, Datum Horizontal WGS 84, Zona 25 Sul, por meio de aparelho GPS Garmim 72, com precisão média de $5 \mathrm{~m}$. Uma amostra por hectare foi adotada por questão de custos e por ser apropriada para trabalho em propriedade rural, conforme Bazzi et al. (2013) e Tavares Filho \& Ribon (2008).

Os talhões que formaram a área experimental deste trabalho apresentam área de 10 ha, com exceção de três deles (Tabela 1). Além disso, entre eles existia variação em relação às datas de plantio e as variedades de cana-deaçúcar cultivadas. Essas variações são inerentes à programação agrícola da empresa, devendo-se ressaltar que, por ocasião das coletas de dados, a cultura estava em início de crescimento para a safra 2012-2013.

A caracterização física do solo da área estudada (Tabela 2) incluiu as variáveis densidade do solo, porosidade total, microporosidade e macroporosidade, determinadas a partir de amostras indeformadas, coletadas em trincheiras com dimensões de $0,50 \times 0,50 \times 0,50 \mathrm{~m}$, escavadas no centro de cada talhão, das quais se retiraram dois anéis por camada, de 0-10, 11-20, 21-30 e 31-40 cm. Também foram determinadas as frações granulométricas do solo e a densidade de partículas. As classes de solo identificadas na área foram Latossolo Amarelo, Argissolo Amarelo e Neossolo Quartzarênico, tendo sido observados impedimentos físicos por cimentação e presença de fragipã $\tilde{a}^{4}$.

A determinação da RSP foi realizada por meio de penetrômetro marca Falker, modelo SoloStar PLG 5500, montado em quadriciclo da marca Shineray, a gasolina, modelo XY 250-STVI. O penetrômetro, dotado de sistema automático de medição de resistência à penetração, foi configurado para resolução da medição de profundidade de $10 \mathrm{~cm}$, tem velocidade nominal de inserção de $3,0 \mathrm{~m} \mathrm{~s}^{-1}$, é alimentado por bateria de $12 \mathrm{~V}$, tem capacidade de memória para 910 medições e usa o protocolo NMEA 0183 de comunicação com GPS. O cone utilizado tem diâmetro de 7,94 mm e, junto com a haste do penetrômetro, segue a norma ASAE S.313.3 (2004). Esse cone permite penetração máxima de $40 \mathrm{~cm}$ de profundidade, índice de cone máximo de 15,1 $\mathrm{MPa}$, com resolução de medição de $0,020 \mathrm{MPa}$ e força máxima suportada na haste de $75 \mathrm{kgf}$.

As variáveis determinadas foram: RSP, média de três leituras para cada ponto amostral, em quatro camadas de $10 \mathrm{~cm}$ de profundidade $\left(\mathrm{RSP}_{0-10} ; \mathrm{RSP}_{11-20} ; \mathrm{RSP}_{21-30} ; \mathrm{RSP}_{31-}\right.$ ${ }_{40}$ ) e a RSP média de 0 a $40 \mathrm{~cm}$, ou Índice de Cone (IC). A cada quatro leituras de RSP, simultaneamente à leitura do penetrômetro, foram retiradas amostras de solo das camadas de 0-10,11-20 e 21-30 cm de profundidade para determinação do teor de água no solo após secagem por 24 horas em estufa, a $105^{\circ} \mathrm{C}$.

As variáveis de resistência do solo à penetração e de teor de água no solo, nas diferentes camadas, foram analisadas por meio da estatística descritiva, sendo calculados média, valor máximo, valor mínimo e coeficiente de variação (CV), classificado por Warrick (1998) como baixo, quando menor do que $15 \%$; médio para $\mathrm{CV}$ entre $15 \mathrm{e}$ $50 \%$ e alto para $\mathrm{CV}$ acima de $50 \%$. O ajuste dos dados à distribuição normal foi verificado pelo Teste Shapiro-Wilk, a 5\% de significância. O teste $t$ para dados pareados foi utilizado para comparar a RSP entre as camadas amostradas.

O teste $t$, a $5 \%$ de significância, foi empregado, do mesmo modo que em Lima et al. (2013b), para verificar diferenças no teor de água do solo entre talhões e entre camadas e, assim, avaliar a necessidade de correção da RSP, em função do teor de água. A análise de correlação entre os dados de RSP e do teor de água também realizada por Dalchiavon et al. (2014) e Carvalho et al. (2008), que utilizaram os coeficientes de correlação de Pearson ou de Spearman, conforme as variáveis ajustaram-se à distribuição normal, ou não.

A estimativa dos valores de RSP para locais não amostrados foi realizada para possibilitar a confecção de mapas de isovalores, por meio de software especializado para modelagem de superfícies. Para isso, foi empregada a interpolação por krigagem, do mesmo modo que em Campos et al. (2013). Esse procedimento, segundo Camargo (1998), considera uma superfície sobre a qual se observa alguma propriedade do solo, $\mathrm{Z}$, em $\mathrm{n}$ pontos distintos, com coordenadas representadas pelo vetor $\mathrm{x}$. Assim, tem-se um conjunto de valores $\{\mathrm{z}(\mathrm{xi}), \mathrm{i}=1, \ldots, \mathrm{n}\}, \mathrm{em}$ que xi identifica uma posição em duas dimensões, representada pelos pares de coordenadas (xi, yi). Supondo-se que se objetive estimar o valor de $Z$ no ponto $X_{0}$, o valor desconhecido de $\mathrm{Z}\left(\mathrm{x}_{0}\right)$ pode ser estimado a partir de uma combinação linear dos $\mathrm{n}$ valores observados, adicionado a um parâmetro, $\lambda_{0}$, conforme a Eq. 1 .

${ }^{4}$ Prado, H do. Identificação e diagnóstico dos solos para a cana-de-açúcar - Grupo Farias. Usina Baía Formosa (RN). Comunicação Pessoal. 7 de outubro de 2011. Instituto Agronômico de Campinas - IAC. 

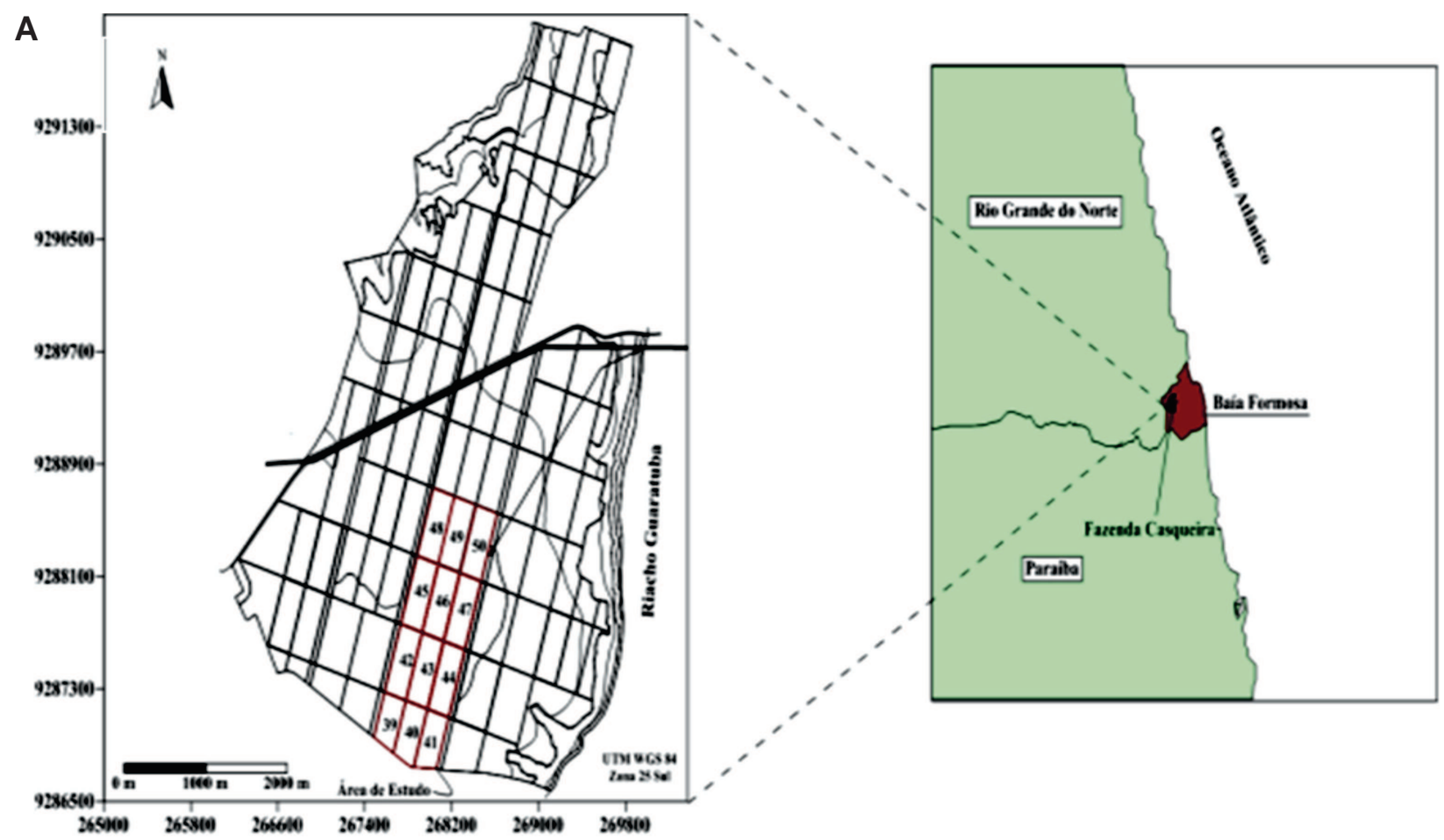

B

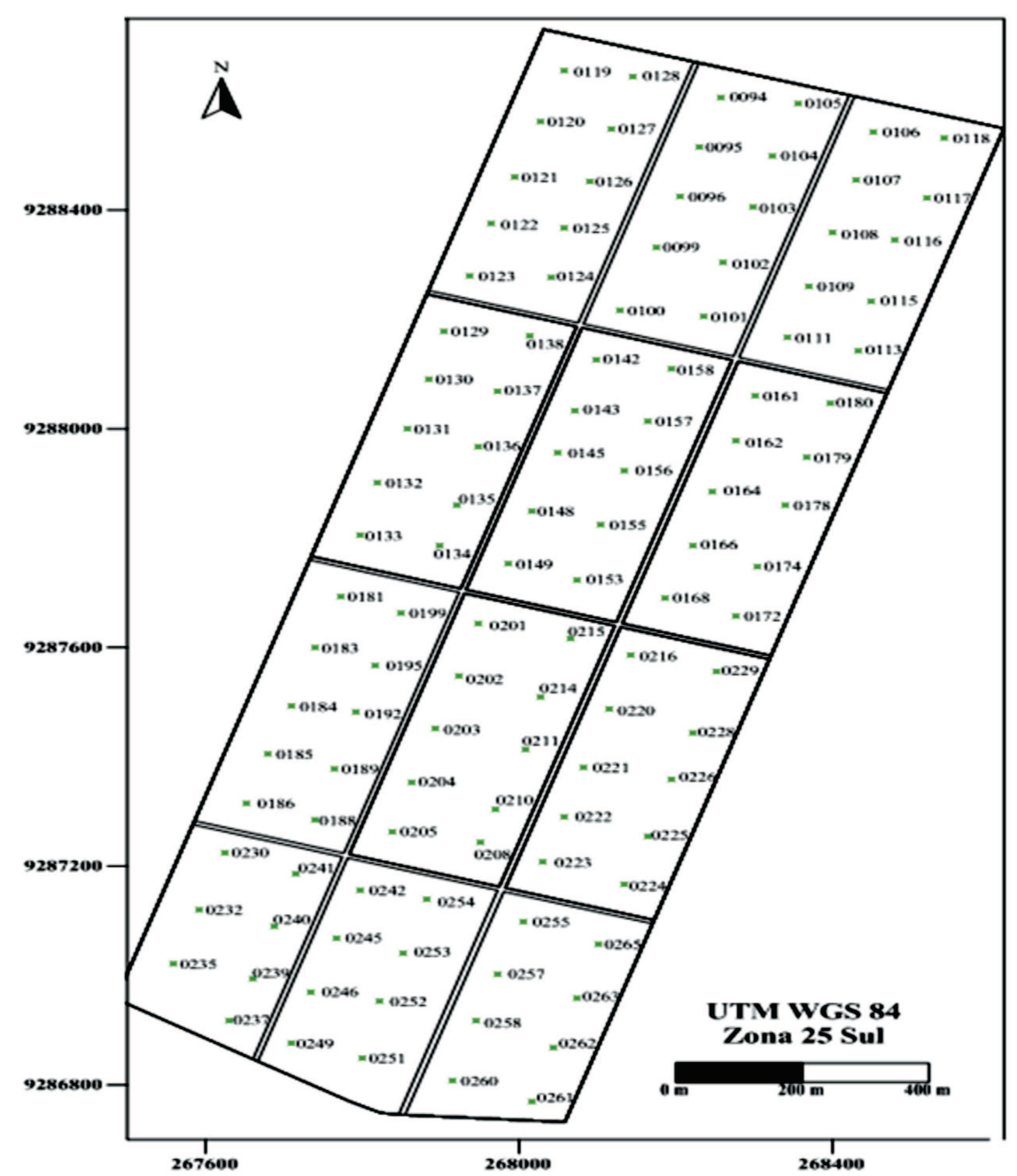

Figura 1: Área da Usina Vale Verde, com a delimitação dos talhões estudados (A) e o Grid dos pontos amostrais georreferenciados (B). Rev. Ceres, Viçosa, v. 63, n.5, p. 715-723, set/out, 2016 
$Z_{X_{0}}^{*}=\lambda_{0}+\sum_{i=1}^{n} \lambda_{i} Z\left(X_{i}\right)$

O critério de validação dos mapas modelados foi a soma dos quadrados dos desvios, considerando-se o valor amostrado frente ao valor modelado em cada ponto amostral, o que é possibilitado pelo software empregado. Desta forma, a interpolação por krigagem sempre apresenta menor resíduo do que outros métodos.

Os níveis de intensidade da resistência do solo à penetração, usados nos mapas modelados, são sugeridos por Ribeiro (2010): de 0 a 2 MPa são baixos e indicam baixa compactação do solo e ausência de impedimento ao crescimento da planta; de 2 a 4 MPa são moderados e indicam o limiar de impedimento ao crescimento da planta; de 4 a 6 MPa são altos e indicam impedimento ao desenvolvimento das raízes; acima de $6 \mathrm{MPa}$ são muito altos. A determinação dos percentuais da área distribuídos em cada nível de intensidade da RSP também foi realizada por meio do software utilizado.

\section{RESULTADOS E DISCUSSÃO}

Os teores de água entre camadas do solo não diferiram pelo teste $t(\mathrm{p}<0,05)$, conforme apresentado, além de que os coeficientes de variação foram médios em todas as camadas, conforme se observa na Tabela 3. A pequena variação nos teores de água do solo indica que os dados obtidos por penetrometria representam efetivamente o estado de compactação do solo e que diferenças em RSP entre locais e camadas não foram devidas a diferenças dos teores de água. Isso concorda com Ribeiro (2010), Molin et al. (2012) e Lima et al. (2013b). A observação de teores de água muito próximos nos locais e camadas amostradas também levou Lima et al. (2013a) e Roque et al. (2010) a ignorarem a influência do teor de água do solo, ao discutir dados de RSP. Isso também foi feito por Gorucu et al (2006), que relataram diferenças significativas dos teores de água, correspondendo a diferenças não significativas em RSP.

Os coeficientes de correlação obtidos (Tabela 4) indicam que, em geral, no momento das medições, não houve

Tabela 1: Caracterização dos talhões de cana-de-açúcar amostrados na Fazenda Casqueira

\begin{tabular}{|c|c|c|c|c|}
\hline Talhões & Área (ha) & Pontos amostrados & Variedades & Data de plantio \\
\hline 39 & 07,20 & 07 & SP832847 & $23 / 07 / 2002$ \\
\hline 40 & 08,31 & 08 & SP832847 & $23 / 07 / 2002$ \\
\hline 41 & 08,08 & 08 & SP832847 & $24 / 07 / 2002$ \\
\hline 42 & 10,00 & 10 & RB867515 & $30 / 05 / 2006$ \\
\hline 43 & 10,00 & 10 & RB962962 & $30 / 01 / 2010$ \\
\hline 44 & 10,00 & 10 & RB931011 & 03/02/2010 \\
\hline 45 & 10,00 & 10 & RB867515 & $30 / 05 / 2006$ \\
\hline 46 & 10,00 & 10 & RB867515 & $13 / 01 / 2010$ \\
\hline 47 & 10,00 & 10 & RB867515 & $12 / 01 / 2010$ \\
\hline 48 & 10,00 & 10 & RB867515 & $31 / 05 / 2006$ \\
\hline 49 & 10,00 & 10 & RB92579 & $09 / 02 / 2010$ \\
\hline 50 & 10,00 & 10 & RB92579 & $25 / 01 / 2010$ \\
\hline Total & 113,59 & 113 & & \\
\hline
\end{tabular}

Fonte: Fazenda Casqueira

Tabela 2: Características físicas do solo da área estudada (médias \pm erro padrão) na Fazenda Casqueira, separadas por camada amostrada

\begin{tabular}{|c|c|c|c|c|}
\hline Profundidade $(\mathrm{cm})$ & Areia $\left(\mathrm{g} \mathrm{kg}^{-1}\right)$ & Silte $\left(\mathrm{g} \mathrm{kg}^{-1}\right)$ & Argila $\left(\mathrm{g} \mathrm{kg}^{-1}\right)$ & Dp $\left(\mathrm{Mg} \mathrm{m}^{-3}\right)$ \\
\hline $0-10$ & $0,89 \pm 0,01$ & $0,03 \pm 0,01$ & $0,07 \pm 0,007$ & $2,51 \pm 0,03$ \\
\hline $11-20$ & $0,88 \pm 0,02$ & $0,04 \pm 0,01$ & $0,07 \pm 0,008$ & $2,46 \pm 0,07$ \\
\hline $21-30$ & $0,87 \pm 0,02$ & $0,04 \pm 0,02$ & $0,09 \pm 0,010$ & $2,43 \pm 0,03$ \\
\hline \multirow[t]{2}{*}{$31-40$} & $0,86 \pm 0,02$ & $0,02 \pm 0,03$ & $0,12 \pm 0,010$ & $2,43 \pm 0,03$ \\
\hline & Ds $\left(\mathrm{Mg} \mathrm{m}^{-3}\right)$ & $P_{\text {tot }}(\%)$ & $\mathrm{MIC}(\%)$ & MAC (\%) \\
\hline $0-10$ & $1,62 \pm 0,021$ & $35,46 \pm 0,67$ & $23,27 \pm 0,70$ & $12,19 \pm 0,59$ \\
\hline $11-20$ & $1,67 \pm 0,022$ & $35,65 \pm 0,58$ & $23,34 \pm 1,04$ & $12,31 \pm 0,73$ \\
\hline $21-30$ & $1,72 \pm 0,019$ & $33,85 \pm 0,77$ & $21,10 \pm 1,22$ & $12,76 \pm 0,85$ \\
\hline $31-40$ & $1,74 \pm 0,038$ & $36,43 \pm 0,61$ & $19,34 \pm 1,46$ & $17,09 \pm 1,49$ \\
\hline
\end{tabular}

$\mathrm{Dp}=$ densidade de partícula; $\mathrm{Ds}=$ densidade do solo; $\mathrm{P}_{\mathrm{tot}}=$ porosidade total $\mathrm{MIC}=$ microporosidade $;$ MAC $=$ macroporosidade 
correlação entre valores de RSP e de teores de água do solo. Por isso, a variação da RSP não pode ser atribuída à variação do teor de água do solo e os dados obtidos não necessitam ser corrigidos em função desse teor. Essas considerações também foram feitas por Dalchiavon et al. (2014) e Carvalho et al. (2008).

As médias da resistência do solo à penetração (Tabela 5) diferiram entre talhões, mas não foi observado aumento da RSP a cada camada consecutiva em todos os talhões; em alguns a RSP diminuiu a partir da camada de $21-30 \mathrm{~cm}$ e, em outros, a diminuição ocorreu a partir da camada de 31-40 $\mathrm{cm}$. Em termos de Índice de Cone (IC0-40), observa-se que a menor média (3,34 MPa) e o menor valor máximo (5,81 $\mathrm{MPa}$ ) ocorreram no talhão 45 e foram pouco inferiores aos do talhão 48 , enquanto a maior média $(6,16 \mathrm{MPa})$ e o valor máximo $(9,97 \mathrm{MPa})$ ocorreram no talhão 47 . O talhão 42 apresentou IC apenas inferior ao do talhão 47, mas sua RSP máxima foi a quinta maior, indicando que a distribuição da compactação difere entre camadas e entre talhões. É possível que esses solos apresentem caráter coeso, caracterizado por serem muito resistentes à penetração e de muito duros a extremamente duros quando secos, como muitos solos cultivados com cana-de-açúcar nos tabuleiros costeiros do nordeste brasileiro (Lima Neto et al., 2009).

Em relação à compactação, Gorucu et al. (2006) descrevem dois tipos de camadas subsuperficiais de solo: as de origem natural, ou adensadas, que apresentam baixa condutividade hidráulica e diferem em propriedades químicas e físicas das camadas adjacentes, e aquelas cuja compactação é devida a pressões externas, que apresentam maiores densidade e porosidade do que as adjacentes. Segundo esses autores, a presença de camada adensada, de origem natural, pode ser indicada por diferenças em textura entre camadas adjacentes de solo. Neste trabalho, isso foi observado apenas na camada de $30-40 \mathrm{~cm}$, que apresentou o maior teor de argila.

A resistência do solo à penetração aumentou com a profundidade (Tabela 3), com diferenças significativas entre camadas pelo teste $\mathrm{t}(\mathrm{p}<0,01)$. Analisando-se por ca- mada, nota-se que a mais superficial $(0-10 \mathrm{~cm})$ era a menos compactada, pois apresentou RSP média de 2,09 MPa, considerada baixa. A partir dela, a RSP passou a ser alta na camada de $11-20 \mathrm{~cm}(5,57 \mathrm{MPa})$ e muito alta entre $21 \mathrm{e}$ $30 \mathrm{~cm}(6,60 \mathrm{MPa})$ e entre 31 e $40 \mathrm{~cm}$ (7,33 MPa), sendo esta a camada mais compactada. Os valores absolutos máximos observados foram semelhantes nas camadas a partir de $10 \mathrm{~cm}$ de profundidade, todos pouco superiores a 13,00 $\mathrm{MPa}$. O ajuste dos dados à normalidade não foi observado para o índice de cone e para os valores de RSP nas camadas de 0-10 e 11-20 cm.

$\mathrm{O}$ aumento dos valores de resistência à penetração com o aumento da profundidade também foi observado por Pereira et al. (2002) em diferentes sistemas de cultivo, o que pode ser característico de solos com alto teor de argila. Nesse sentido, os valores de resistência à penetração obtidos por Lima et al. (2013b) em solo de textura franco-argilo-arenosa foram maiores do que em solos franco-arenoso e muito argiloso, os quais não diferiram. Em solo argilo-arenoso de área experimental cultivada nos últimos 15 anos, Lima et al. (2013a) obtiveram 1,94 MPa como maior valor de RSP, por meio de dois tipos de penetrômetros, muito abaixo dos valores obtidos neste trabalho.

A variabilidade dos dados é demonstrada pelas amplitudes elevadas de valores e pelos coeficientes de variação (Tabela 4). As camadas de 0-10 (61,19\%) e de $11-20 \mathrm{~cm}$ $(55,45 \%)$ apresentaram CV alto. Tavares Filho \& Ribon

Tabela 4: Coeficientes de correlação entre resistência do solo à penetração e teores de água do solo nas diferentes camadas

\begin{tabular}{lccc}
\hline & $\begin{array}{c}\text { Teor de } \\
\text { água 0-10 }\end{array}$ & $\begin{array}{c}\text { Teor de } \\
\text { água 10-20 }\end{array}$ & $\begin{array}{c}\text { Teor de } \\
\text { água 20-30 }\end{array}$ \\
\hline RP 0-10 & $-0,18^{\text {ns }}$ & $-0,014^{\text {ns }}$ & $0,23^{\text {ns }}$ \\
RP 11-20 & $-0,16^{\text {ns }}$ & $0,011^{\text {ns }}$ & $-0,15^{\text {ns }}$ \\
RP 21-30 & $-0,33^{\text {ns }}$ & $-0,068^{\text {ns }}$ & $-0,36^{\text {ns }}$ \\
RP 31-40 & $-0,12^{\text {ns }}$ & $0,147^{\text {ns }}$ & $-0,41^{*}$ \\
IC 0-40 & $-0,28^{\text {ns }}$ & $-0,020^{\text {ns }}$ & $-0,31^{\text {ns }}$ \\
\hline
\end{tabular}

${ }^{\mathrm{n} s}$ ão significativo; *significativo a $5 \%$ de probabilidade.

Tabela 3: Estatística descritiva da resistência do solo à penetração e do teor de água no solo das diferentes camadas amostradas na área de estudo

\begin{tabular}{|c|c|c|c|c|c|}
\hline Variável & Média & Mínimo & Máximo & ${ }^{2} \mathrm{CV}(\%)$ & ${ }^{3} \mathbf{S W}$ \\
\hline${ }^{1} \mathrm{RP}_{0-10}(\mathrm{MPa})$ & $2,08 \mathrm{~d}$ & 0,08 & 05,64 & 61,19 & 0,0001 \\
\hline $\mathrm{RP}_{10-20}(\mathrm{MPa})$ & $5,57 \mathrm{c}$ & 1,04 & 13,77 & 55,45 & 0,0001 \\
\hline $\mathrm{RP}_{20-30}(\mathrm{MPa})$ & $6,60 \mathrm{~b}$ & 1,68 & 13,38 & 38,17 & 0,0800 \\
\hline $\mathrm{RP}_{30-40}(\mathrm{MPa})$ & $7,33 \mathrm{a}$ & 1,68 & 13,94 & 34,08 & 0,3700 \\
\hline $\mathrm{IC}_{0-40}(\mathrm{MPa})$ & 5,21 & 1,68 & 09,88 & 32,85 & 0,0042 \\
\hline Teor de água $_{0-10}(\%)$ & $5,53 \mathrm{a}$ & 1,42 & 12,58 & 48,03 & 0,1900 \\
\hline Teor de água ${ }_{10-20}(\%)$ & $6,07 \mathrm{a}$ & 1,84 & 11,73 & 39,73 & 0,1100 \\
\hline Teor de água ${ }_{20-30}(\%)$ & $6,73 \mathrm{a}$ & 1,80 & 12,64 & 38,61 & 0,3900 \\
\hline
\end{tabular}

${ }^{1}$ Resistência à penetração; ${ }^{2}$ Coeficiente de variação; ${ }^{3}$ Significância do teste Shapiro Wilk= ajustam-se à distribuição normal as variáveis com Pr>0,05; médias de camadas seguidas de letras diferentes diferem pelo teste t para dados pareados a $1 \%$ de significância. 
(2008) e Ribeiro (2010) também encontraram os maiores valores de CV, 42,85 e 54,43\%, respectivamente, na camada superficial de Latossolos. Os CV médios das camadas de $21-30(38,17 \%)$ e $31-40 \mathrm{~cm}(34,08 \%)$ concordam com os resultados obtidos por Souza et al. (2006).

Os mapas bidimensionais de isovalores (Figura 2) permitem visualizar o comportamento espacial dos valores de resistência do solo à penetração nas diversas camadas e em termos médios (IC), além de que o referenciamento das regiões de interesse permite sua verificação in loco. A importância desses mapas está na possibilidade de empregá-los para planejar ações de manejo localizado, como a variação na profundidade do preparo do solo ao longo da área, segundo a intensidade de compactação em cada região do campo, conforme foi estudado por Gorucu et al. (2006).

A escolha da densidade de amostragem é importante para o sucesso do manejo sítio-específico, devendo seguir critérios de ordem econômica e prática e representar adequadamente as diferentes regiões do campo e os diferentes níveis de seus atributos. Nesse aspecto, a adoção de uma amostra por hectare, ou menos, é comum em áreas sob agricultura de precisão no Brasil (Franzen \& Peck, 1995; Cherubin et al., 2014). Em trabalhos relacionados com a cana-de-açúcar, Pellin et al (2015) adotaram a mesma densidade amostral, enquanto Lima et al (2013b) usa- ram malha ainda menos densa. A mesma malha amostral adotada neste trabalho captou as diferentes escalas de variabilidade espacial dos atributos, em estudo de Cherubin et al. (2014), enquanto a opção entre malhas de amostragem de 67 x 67 m ou de 100 x 100 m, em estudo de Franzen \& Peck (1995), dependeria da maior ou menor variabilidade do campo.

Os elevados valores de RSP obtidos neste trabalho podem ser devidos a propriedades do solo, como teor de água e de matéria orgânica, a operações mecanizadas e ao tráfego de máquinas, os quais acarretam diferenças na resistência mecânica ao longo do perfil. A relação entre os altos valores de RSP deste trabalho e os teores muito baixos de água no solo, no momento das medições, é corroborada por Molin et al. (2012) que, no momento das suas medições de RSP, observaram teores de água abaixo dos considerados ideais, os quais seriam de $2 / 3$ da microporosidade do solo, segundo Dalchiavon et al.(2014).

As operações agrícolas que mobilizam o solo e o tráfego de máquinas alteram a estrutura do solo e modificam as condições para o crescimento radicular. A pressão dos equipamentos provoca a propagação em profundidade da força exercida, de maneira que a resposta do solo se dá sob forma de diferentes valores de resistência mecânica, ao longo do perfil, sendo altamente dependente da carga aplicada (Reichert et al., 2007). Atualmente, a colheita me-

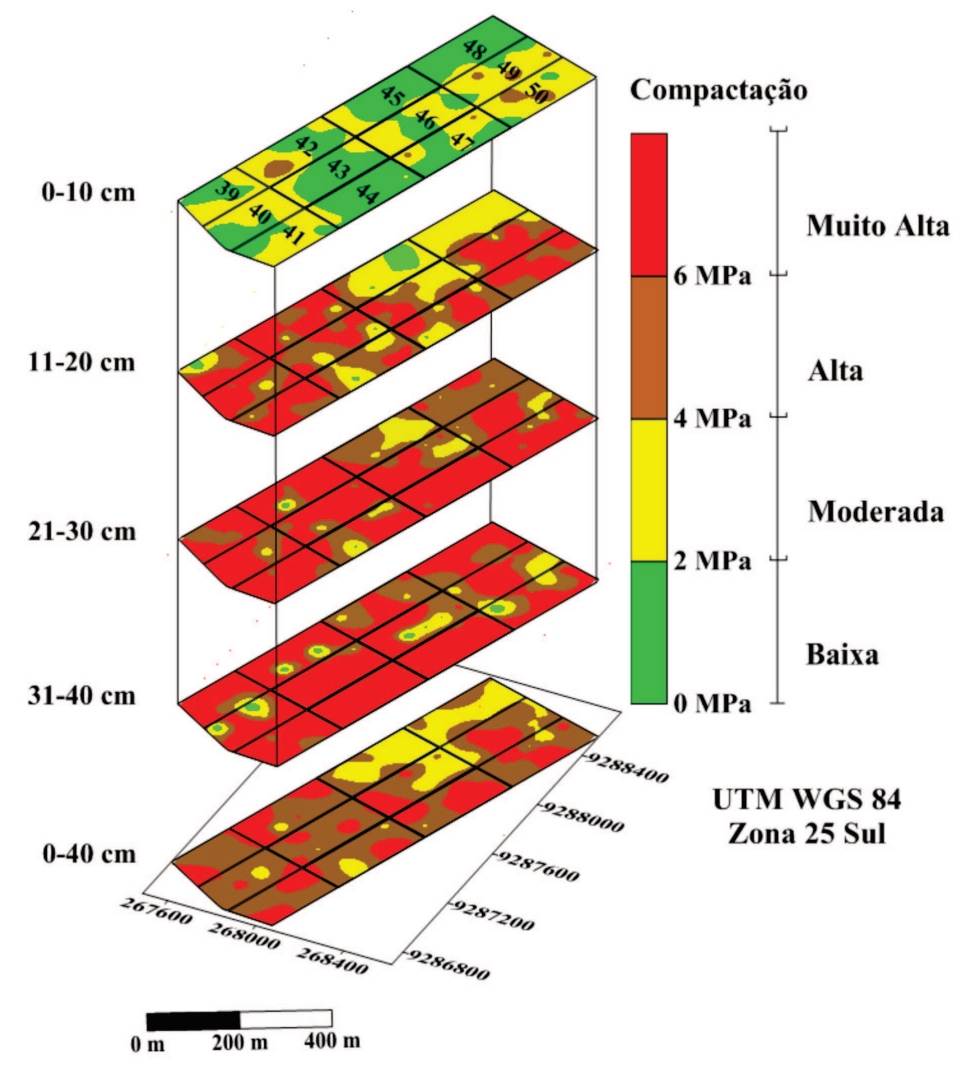

Figura 2: Espacialização dos valores críticos da resistência do solo à penetração (RSP) e classificação dos níveis de compactação do solo, referente a cada uma das camadas do solo para 12 talhões da área experimental. 
canizada da cana-de-açúcar, quando o solo apresenta teores elevados de água, é apontada como causa importante da compactação do solo, principalmente nos primeiros 30 a $40 \mathrm{~cm}$ de profundidade (Severiano et al., 2010).

A análise da RSP por camada (Figura 2) permite observar que a camada mais superficial $(0-10 \mathrm{~cm})$ apresenta predominância de baixa RSP (até $2 \mathrm{MPa}$ ), seguida da moderada (de 2 a $4 \mathrm{MPa}$ ). À medida que se analisam camadas mais profundas, observa-se que maiores intensidades de compactação passam a predominar, como moderada e muito alta (a partir de $6 \mathrm{MPa}$ ), na camada de 11 a $20 \mathrm{~cm}$, muito alta e alta (4 a $6 \mathrm{MPa}$ ), de 21 a $30 \mathrm{~cm}$, e muito alta, de 31 a $40 \mathrm{~cm}$. Nesse aspecto, os mapas de isovalores apresentam intensidades menores de compactação em todas as camadas da região, englobando os talhões 45, 46 e 48 que, por isso, poderiam receber preparo menos intenso.

Considerando-se que os efeitos da compactação sobre uma cultura resultam do comportamento da resistência do solo em toda a camada explorada pelas raízes, o mapa de isovalores da RSP média de 0 a $40 \mathrm{~cm}$ indica que, nos talhões 45, 46 e 48, a cultura teria melhores condições de desenvolvimento radicular e, por isso, melhor produção. Além disso, observa-se que a alta compactação pre- domina no campo, diferentemente do observado em cada camada. Os resultados deste trabalho são bem superiores aos obtidos por Campos et al. (2013), que avaliaram a variabilidade espacial da RSP e do teor de água do solo em área cultivada com cana-de-açúcar e encontraram 2,86 MPa como o maior valor de RSP, que ocorreu na profundidade de 0,15-0,30 m.

Outro aspecto importante para o manejo dos solos compactados é o conhecimento das proporções da área do campo afetada pelas diferentes intensidades de compactação. Nesse aspecto, verifica-se que na camada de $0-10 \mathrm{~cm}$ de profundidade predomina a RSP baixa, presente em 58,54\% da área, mas também existem $45,30 \%$ de áreas com níveis moderados e uma pequena área com níveis altos (Tabela 6). Enquanto isso, nas camadas mais profundas predomina a compactação muito alta, chegando a 69,00\% da área na camada de $31-40 \mathrm{~cm}$, e pequena área com compactação baixa. Entretanto, quando se analisa a RSP média de todo perfil, a compactação alta predomina em 55,56\% da área. Essas proporções podem indicar a viabilidade de se adotarem práticas mais específicas de manejo, em termos de custo ou de tempo para sua realização.

Tabela 5: Valores médios da resistência do solo à penetração (RSP) por talhão

\begin{tabular}{lcccccc}
\hline \multirow{2}{*}{ Talhão } & \multicolumn{7}{c}{ Resistência do solo à penetração - RSP (MPa) } \\
\cline { 2 - 7 } & $\mathbf{0}$ & \multicolumn{7}{c}{ Profundidades (cm) } \\
\cline { 2 - 7 } & $\mathbf{0 - 1 0}$ & $\mathbf{1 0 - 2 0}$ & $\mathbf{2 0 - 3 0}$ & $\mathbf{3 0 - 4 0}$ & $\mathbf{0 - 4 0 * *}$ & Valor máximo \\
\hline 39 & 2,27 & 6,47 & 7,08 & 7,71 & 5,48 & 7,75 \\
40 & 2,43 & 7,75 & 8,03 & 5,31 & 5,50 & 9,45 \\
41 & 2,65 & 7,16 & 7,73 & 7,56 & 5,87 & 7,83 \\
42 & 2,73 & 7,84 & 7,54 & 7,34 & 5,92 & 8,20 \\
43 & 1,54 & 5,92 & 7,56 & 8,44 & 5,40 & 9,72 \\
44 & 1,53 & 4,99 & 6,03 & 8,79 & 4,91 & 9,00 \\
45 & 1,28 & 2,95 & 4,49 & 5,67 & 3,34 & 5,81 \\
46 & 2,30 & 4,77 & 6,26 & 6,57 & 4,68 & 7,16 \\
47 & 2,23 & 6,70 & 8,40 & 9,31 & 6,16 & 9,79 \\
48 & 0,98 & 3,42 & 5,74 & 6,40 & 3,74 & 6,78 \\
49 & 3,16 & 6,30 & 5,95 & 6,03 & 5,11 & 6,80 \\
50 & 3,00 & 7,27 & 6,91 & 5,15 & 5,30 & 7,57 \\
\hline Média Geral & 2,08 & 5,57 & 6,60 & 7,33 & 5,02 & 7,99 \\
\hline
\end{tabular}

** IC=Índice de cone

Tabela 6: Distribuição em hectares e em percentagem da área dos intervalos da resistência do solo à penetração (RSP)

\begin{tabular}{|c|c|c|c|c|c|c|c|c|}
\hline \multirow{3}{*}{ Profundidade $(\mathrm{cm})$} & \multicolumn{8}{|c|}{ Intervalos da RSP (MPa) } \\
\hline & \multicolumn{2}{|c|}{0 a 2} & \multicolumn{2}{|c|}{2 a 4} & \multicolumn{2}{|c|}{4 a 6} & \multicolumn{2}{|c|}{ Acima de 6} \\
\hline & (ha) & $(\%)$ & (ha) & $(\%)$ & (ha) & $(\%)$ & (ha) & $(\%)$ \\
\hline $0-10$ & 58,54 & 51,28 & 51,47 & 45,30 & 3,87 & 3,40 & 0,00 & 0,00 \\
\hline $11-20$ & 2,27 & 2,00 & 27,26 & 24,00 & 37,48 & 33,00 & 46,57 & 41,00 \\
\hline $21-30$ & 0,96 & 0,85 & 7,77 & 6,84 & 35,92 & 31,62 & 68,94 & 60,69 \\
\hline $31-40$ & 1,94 & 1,71 & 6,79 & 5,98 & 26,22 & 23,08 & 78,64 & 69,23 \\
\hline $0-40$ & 0,00 & 0,00 & 21,35 & 18,80 & 63,11 & 55,56 & 29,12 & 25,64 \\
\hline
\end{tabular}

Rev. Ceres, Viçosa, v. 63, n.5, p. 715-723, set/out, 2016 


\section{CONCLUSÕES}

O georreferenciamento localiza no campo áreas com diferentes níveis de compactação do solo.

Foram identificados $18,80 \%$ da área total nos quais a descompactação do solo pode ser evitada, principalmente nos talhões.

Nas áreas identificadas como necessitando descompactação, esta deve ser realizada a profundidade maior do que $40 \mathrm{~cm}$.

\section{REFERÊNCIAS}

ASAE - American Society of Agricultural Engineers (2004) Soil cone penetrometer - ASAE Standard S313.3. Saint Joseph, ASAE. $3 p$.

Bazzi CL, Souza EG, Uribe-Opazo MA, Nóbrega LHP \& Rocha DM (2013) Management zones definition using soil chemical and physical attributes in a soybean area. Engenharia Agrícola, 34:952-964.

Bianchini A, Sabino MHC, Borges PHM \& Sguarezzi JJ (1999) Comportamento operacional de um escarificador de Hastes parabólicas em solo de cerrado. Revista Brasileira de Engenharia Agrícola e Ambiental, 3:395-401.

Camargo ECG (1998) Geoestatística: fundamentos e aplicações. In: Câmara G \& Medeiros JS (Eds.) Geoprocessamento para projetos ambientais. São José dos Campos, INPE. p.1-36.

Campos MCC, Aquino RE, Oliveira IA \& Bergamim AC (2013) Variabilidade espacial da resistência mecânica do solo à penetração e umidade do solo em área cultivada com cana-de-açúcar na região de Humaitá, Amazonas, Brasil. Agrária, 8:305-310.

Carvalho LA, Neto VJM, Silva LF, Pereira JG, Nunes WAGA \& Chaves CHC (2008) Resistência mecânica do solo à penetração (RMP) sob cultivo de cana-de-açúcar, no município de Rio Brilhante-MS. Agrarian, 1:7-22.

Cherubin MR, Santi AL, Eitelwein MT, Menegol DR, Da Ros CO, Pias OHC \& Berghetti J (2014) Eficiência de malhas amostrais utilizadas na caracterização da variabilidade espacial de fósforo e potássio. Ciência Rural, 44:425-432.

Dalchiavon FC, Carvalho MP, Montanari R, Andreotti M \& Dal Bem EA (2014) Inter-relações da produtividade de cana soca com a resistência à penetração, umidade e matéria orgânica do solo. Revista Ceres, 61:255-264.

Franzen DW \& Peck TR (1995) Field soil sampling density for variable rate fertilization. Journal of Production Agriculture, $8: 568-574$.

Gorucu S, Khalilian A, Han YJ, Dodd RB \& Smith BR (2006) An algorithm to determine the optimum tillage depth from soil penetrometer data in coastal plain soils. Applied Engineering in Agriculture, 22:625-631.

Gorucu Keskin S, Khalilian A, Han YJ \& Dodd RB (2011) Variabledepth tillage based on geo-referenced soil compaction data in coastal plain soils. International Journal of Applied Science and Technology, 1: 22-32.

IBGE - Instituto Brasileiro de Geografia e Estatística (2014) Produção agrícola municipal. Culturas temporárias e permanentes. Produção Agrícola Municipal, 41:1-100.

Lima RP, Leon MJ \& Silva AR (2013a) Comparação entre dois penetrômetros na avaliação da resistência mecânica do solo à penetração. Revista Ceres, 60:577-581
Lima RP, Leon MJ \& Silva AR (2013b) Compactação do solo de diferentes classes texturais em áreas de produção de cana-deaçúcar. Revista Ceres, 60:16-20.

Lima Neto JA, Ribeiro MR, Corrêa MM, Souza Júnior VS, Lima JFWF \& Ferreira RFAL (2009) Caracterização e gênese do caráter coeso em latossolos amarelos e argissolos dos tabuleiros costeiros do estado de alagoas. Revista Brasileira de Ciência do Solo, 33:1001-1011.

Molin JP, Dias CTS \& Carbonera L (2012) Estudos com penetrometria: Novos equipamentos e amostragem correta. Revista Brasileira de Engenharia Agrícola e Ambiental, 16:584590 .

Pellin DMP, Rafael Montanari, Elizeu de Souza Lima, Lenon Henrique Lovera \& Adriany Rodrigues Corrêa (2015) Variabilidade de atributos físicos de um latossolo vermelho sob cultivo de cana-de-açúcar. Caatinga, 28: 28-38.

Pereira JO, Siqueira JAC, Uribe-Opazo MA \& Silva SL (2002) Resistência do solo à penetração em função do sistema de cultivo e teor de água do solo. Revista Brasileira de Engenharia Agrícola e Ambiental, 6:171-174.

Raper RL, Reeves DW, Shaw JN, Van Santen E \& Mask PL (2007) Benefits of site-specific subsoiling for cotton production in Coastal Plain soils. Soil \& Tillage Research, 96:174-181.

Reichert JM, Suzuki LEAS \& Reinert DJ (2007). Compactação do solo em sistemas agropecuários e florestais: identificação, efeitos, limites críticos e mitigação. In: Ceretta CA, Silva LS \& Reichert JM (Eds.) Tópicos em ciência do solo. Viçosa, Sociedade Brasileira de Ciência do Solo. p.49-134.

Ribeiro CA (2010) Variabilidade espacial da resistência mecânica do solo à penetração em áreas mecanizadas de cana-de-açúcar (Saccharum officinarum). Dissertação de Mestrado. Universidade Estadual Paulista, Jaboticabal. 85p.

Rodrigues LD (2010) A cana-de-açúcar como matéria-prima para a produção de biocombustíveis: impactos ambientais e o zoneamento agroecológico como ferramenta para mitigação. Trabalho de conclusão de curso. Faculdade de Engenharia, Universidade Federal de Juiz de Fora, Juiz de Fora. 64p.

Roque AAO, Souza ZM, Barbosa RS \& Souza GS (2010) Controle de tráfego agrícola e atributos físicos do solo em área cultivada com cana-de-açúcar. Pesquisa Agropecuária Brasileira, 45:744750 .

Salvador N, Benez SH \& Mion RL (2008) Consumo de combustível na operação de subsolagem realizada antes e depois de diferentes sistemas de preparo periódico do solo. Engenharia Agrícola, 28:256-262.

Severiano EC, Oliveira GC, Dias Júnior MS, Castro MB, Oliveira LC \& Costa KAP (2010) Compactação de solos cultivados com cana-de-açúcar: I - modelagem e quantificação da compactação adicional após as operações de colheita. Engenharia Agrícola, 30:404-413. 